\title{
PSEUDOBASES IN DIRECT POWERS OF AN ALGEBRA
}

\author{
PAUL BANKSTON
}

\begin{abstract}
A subset $P$ of an abstract algebra $A$ is a pseudobasis if every function from $P$ into $A$ extends uniquely to an endomorphism on $A$. $A$ is called $\kappa$-free if $A$ has a pseudobasis of cardinality $\kappa ; A$ is minimally free if $A$ has a pseudobasis. (The 0 -free algebras are "rigid" in the strong sense; the 1-free groups are always abelian, and are precisely the additive groups of $E$-rings.) Our interest here is in the existence of pseudobases in direct powers $A^{I}$ of an algebra $A$. On the positive side, if $A$ is a rigid division ring, $\kappa$ is a cardinal, and there is no measurable cardinal $\mu$ with $|A|<\mu \leq \kappa$, then $A^{I}$ is $\kappa$-free whenever $|I|=\left|A^{\kappa}\right|$. On the negative side, if $A$ is a rigid division ring and there is a measurable cardinal $\mu$ with $|A|<\mu \leq|I|$, then $A^{I}$ is not minimally free.
\end{abstract}

\section{INTRODUCTION}

A pseudobasis in an abstract algebra is a subset of the underlying set of the algebra that "determines" the algebra's endomorphism structure, in the sense that functions from the pseudobasis into the underlying set extend uniquely to endomorphisms of the algebra. Algebras possessing pseudobases are termed minimally free.

The notion of minimal freeness, first introduced in [1], generalizes (and therefore unifies) the apparently unrelated theories of free algebras (which have pseudobases that are generating sets), rigid algebras (which have empty pseudobases), and $E$-rings (which are the endomorphism rings of groups having singleton pseudobases). (Such groups are easily shown [1] to be abelian.) Since its inception, the study of minimal freeness has served as a link between universal algebra and infinitary combinatorics, and in addition has motivated results in general topology through the investigation of minimally free rings of continuous real-valued functions $($ see $[4,5])$.

In this paper we consider the problem of when a direct power of an algebra is minimally free. In the first section we obtain some general results, and in the remaining two sections, our study is carried to the setting of division rings and Boolean rings. We consider the main result of the paper to be 2.10 , a "straddling-a-measurable-cardinal" theorem.

Received by the editors November 30, 1989.

1980 Mathematics Subject Classification (1985 Revision). Primary 03C05, 08E55, 08A35, 08B20, 12L10, 16A39.

Key words and phrases. Pseudobases, direct powers, universal algebra, minimally free, fields, division rings, measurable cardinals. 


\section{Powers of General Algebras}

Let $\Omega$ be a set of finitary operation symbols in the sense of universal algebra $[6,11]$. For any $n<\omega$ (where $\omega$ is the first infinite ordinal), an $n$-place symbol from $\Omega$ is termed $n$-ary; $n$ is called the arity of the symbol. (0-ary symbols are called constants.) An interpretation $\nu_{A}$ of an operation symbol $\nu \in \Omega$ of arity $n$ in a set $A$ is just a map from the $n$-fold cartesian power $A^{n}$ of $A$ into $A$; an $\Omega$-algebra is a set $A$ together with an interpretation in $A$ for each symbol in $\Omega$. We benignly confuse notation by letting upper-case Latin letters $A, B, \ldots$ stand for both $\Omega$-algebras and their underlying sets.

An $\Omega$-algebra $A$ is minimally free if there is a subset $P \subseteq A$, called a pseudobasis, such that every function from $P$ into $A$ extends uniquely to an endomorphism on $A$. (For two $\Omega$-algebras $A$ and $B, \operatorname{Hom}(A, B)$ is the set of all ( $\Omega$-) homomorphisms from $A$ to $B ; \operatorname{End}(A)=\operatorname{Hom}(A, A)$ is the set of endomorphisms on $A$.) If $A$ has a pseudobasis of cardinality $\kappa, A$ is termed $\kappa$-free $[1,2,3,4,5,12]$. (Thus the 0 -free $\Omega$-algebras are precisely the (endomorphism-) rigid ones. A group $A$ is 1 -free if and only if $A$ is the additive group of an $E$-ring [1, 3, 9]. Of course a free algebra in any variety is minimally free.)

Given an $\Omega$-algebra $A$ and a nonempty set $I$, the direct power is denoted $A^{I}$, and consists of all functions $f: I \rightarrow A . A^{I}$ is made into an $\Omega$-algebra by defining the operations pointwise as usual. For each $i \in I$, the ith projection map is denoted $\pi_{i} ; \pi_{i}(f)=f(i)$. Then of course we have $\pi_{i} \in \operatorname{Hom}\left(A^{I}, A\right)$, $i \in I$. The diagonal map $\delta: A \rightarrow A^{I}$ takes $a \in A$ to the "constantly $a$ " map in $A^{I}$. The image $\delta(A)$ is also denoted generically by $\Delta$. Clearly $\delta \in$ $\operatorname{Hom}\left(A, A^{I}\right)$, and $\pi_{i} \circ \delta=\operatorname{id}_{A}$, the identity map on $A$. Thus $\Delta$ is always an isomorphic copy of $A$ that sits in $A^{I}$ as a retract.

If $\kappa$ is a cardinal and $I=A^{\kappa}$, we denote the power $A^{I}$ by $A \uparrow \kappa$ (for obvious typographical reasons). We call this algebra the $\kappa$-fold double power of $A$. (Of course we identify $A \uparrow 0$ with $A$ and $A \uparrow 1$ with $A^{A}$.) An important subset of $A \uparrow \kappa$ is the set $\Pi$ of projection maps $\pi_{\xi}: A^{\kappa} \rightarrow A$ for $\xi<\kappa$. When $A$ is nontrivial, i.e., when the cardinality $|A|$ of $A$ is at least $2, \Pi$ has cardinality $\kappa$. We are interested in the issue of when $\Pi$ is a pseudobasis for $A \uparrow \kappa$, making the double power $\kappa$-free.

Define a subset $P$ of an $\Omega$-algebra $A$ to be extendible if every function from $P$ to $A$ extends (possibly not uniquely) to an endomorphism on $A$. Deciding whether a given subset of $A^{I}$ is extendible or a pseudobasis is facilitated by the following.

1.1 Lemma. Let $P \subset A^{I}$. Then $P$ is an extendible subset (resp. a pseudobasis) if and only if every function from $P$ to $A$ extends (resp. extends uniquely) to a homomorphism from $A^{I}$ to $A$.

Proof. Suppose $P \subseteq A^{I}$ is a pseudobasis, with $f: P \rightarrow A$. Let $\psi \in \operatorname{End}\left(A^{I}\right)$ extend $\delta \circ f$. Pick $i \in I$ and let $\varphi=\pi_{i} \circ \psi$. Then clearly $\varphi \in \operatorname{Hom}\left(A^{I}, A\right)$ extends $f$, so $\delta \circ \varphi$ extends $\delta \circ f$. $\psi$ is unique, so $\delta \circ \varphi=\psi$. If $\varphi^{\prime}=$ $\operatorname{Hom}\left(A^{I}, A\right)$ were another extension of $f$, we would have $\delta \circ \varphi^{\prime}=\psi=\delta \circ \varphi$; hence $\varphi^{\prime}=\varphi$.

Conversely, suppose every function from $P$ to $A$ extends uniquely to a homomorphism from $A^{I}$ to $A$, and let $f: P \rightarrow A^{I}$ be given. For each $i \in I$, let $f_{i}=\pi_{i} \circ f$, with $\varphi_{i} \in \operatorname{Hom}\left(A^{I}, A\right)$ the extension of $f_{i}, i \in I$. Let 
$\psi \in \operatorname{End}\left(A^{I}\right)$ be defined by the coordinate conditions $\pi_{i} \circ \psi=\varphi_{i}$. Then $\psi$ is the unique extension of $f$ to $\operatorname{End}\left(A^{I}\right)$.

1.2 Proposition. $\Pi$ is a pseudobasis for $A \uparrow \kappa$ if and only if every homomorphism from $A \uparrow \kappa$ to $A$ is determined by its restriction $\varphi \mid \Pi$ to $\Pi$.

Proof. By 1.1, $\Pi$ is extendible; since if $f: \Pi \rightarrow A$ is given, the map $\varphi: A \uparrow \kappa$ $\rightarrow A$, defined by $\varphi(s)=s\left(\left\langle f\left(\pi_{\xi}\right): \xi<\kappa\right\rangle\right), s \in A \uparrow \kappa$, is a homomorphism extending $f$.

1.3 Remarks. (i) Any extendible subset of an algebra is "independent" in the sense of E. Marczewski [11].

(ii) Any pseudobasis is a set of "indiscernibles" in the model-theoretic sense [7]. (See also [3] for a simple proof.)

(iii) As was discovered by G. Birkhoff [6], $\Pi$ is a pseudobasis for the subalgebra $\langle\Pi\rangle$ of $A \uparrow \kappa$ generated by $\Pi$. In fact, pseudobases that generate are free bases [11].

(iv) Theorem 2 in [2] says that if $A$ is a rigid algebra, then $\Pi$ is a pseudobasis for $\langle\Pi \cup \Delta\rangle$ in $A \uparrow \kappa$. Thus pseudobases can fail dramatically to be generating sets.

There is a kind of converse to 1.3(iv): It is " almost always" the case that $A$ must be rigid for $\Pi$ to be a pseudobasis for some subalgebra of $A \uparrow \kappa$ containing $\Pi \cup \Delta$.

1.4 Lemma. Suppose $\Omega$ contains a constant symbol $c$. If $A$ is an $\Omega$-algebra, $I$ is a nonempty set, $B$ is a subalgebra of $A^{I}$ that contains $\Delta, Q \subseteq B$ is such that $\bigcap_{q \in Q} q^{-1}\left(c_{A}\right) \neq \varnothing$, and every $\varphi \in \operatorname{Hom}(B, A)$ is determined by its restriction to $Q$, then $A$ is rigid.

Proof. Suppose $A$ is not rigid, with $B$ and $Q$ as above. Let $\varphi, \psi \in \operatorname{End}(A)$ disagree at $a \in A$, let $i \in \bigcap_{q \in Q} q^{-1}\left(c_{A}\right)$, and set $\varphi^{\prime}=\varphi \circ\left(\pi_{i} \mid B\right), \psi^{\prime}=$ $\psi \circ\left(\pi_{i} \mid B\right)$. For each $q \in Q$, we have $\varphi^{\prime}(q)=\varphi(q(i))=\varphi\left(c_{A}\right)=c_{A}=\psi\left(c_{A}\right)=$ $\psi^{\prime}(q)$. Thus $\varphi^{\prime}$ and $\psi^{\prime}$ agree on $Q$. However $\varphi^{\prime}(\delta(a))=\varphi(a) \neq \psi(a)=$ $\psi^{\prime}(\delta(a))$. Thus $\varphi^{\prime}, \psi^{\prime} \in \operatorname{Hom}(B, A)$ are distinct.

1.5 Proposition. Suppose $\Omega$ contains a constant symbol, $B$ is a subalgebra of $A \uparrow \kappa$ containing $\Pi \cup \Delta$, and $\Pi$ is a pseudobasis for $B$. Then $A$ is rigid.

Proof. For any $a \in A, \bigcap_{\xi<\kappa} \pi_{\xi}^{-1}(a)=\{\delta(a)\}$. Apply 1.4.

It can easily happen that $\Omega$ contains a constant, $A \uparrow \kappa$ is minimally free, but that $\Pi$ is not a pseudobasis and $A$ is not rigid. (E.g., let $\Omega=\{c\}$, where $c$ is a constant symbol. Then every $\Omega$-algebra has a pseudobasis (the complement of the interpretation of $c$ ), but only the trivial $\Omega$-algebra is rigid.) We do not know in general whether $\Pi$ is a pseudobasis for $A \uparrow \kappa$ given that $A \uparrow \kappa$ is $\kappa$-free; however if both $A$ and $\kappa$ are finite, the answer is yes.

1.6 Proposition. Suppose $A$ is a finite $\Omega$-algebra and $n<\omega$. If $A \uparrow n$ is $n$-free, then $\Pi$ is a pseudobasis.

Proof. Let $P \subseteq A \uparrow n$ be a pseudobasis with $n$ elements, and let $f: P \rightarrow \Pi$ be a bijection. Let $\varphi \in \operatorname{End}(A \uparrow n)$ extend $f$. Since $\Pi$ is extendible, there is some $\psi \in \operatorname{End}(A \uparrow n)$ extending $f^{-1}$. Now $(\psi \circ \varphi) \mid P=\operatorname{id}_{P}$. Since $P$ is a pseudobasis, we know $\psi \circ \varphi=\operatorname{id}_{A \uparrow n}$; whence $\varphi$ is one-one. Since $A \uparrow n$ is 
finite, $\varphi$ is also onto. Hence $\varphi$ is an automorphism on $A \uparrow n$ taking $P$ to $\Pi$, so $\Pi$ is a pseudobasis.

In $\S 2$ we take up the issue of finding conditions sufficient for $\Pi$ to be a pseudobasis for $A \uparrow \kappa$. For the remainder of this section, we examine the role of pseudobases generally in direct powers. Our working set theory consists of the usual Zermelo-Fraenkel axioms, the Axiom of Choice included (ZFC). The following standard notations for operations on cardinal numbers are used: (i) $\kappa^{+}$is the cardinal successor of $\kappa\left(\omega_{1}=\omega^{+}\right)$; (ii) $\kappa^{\lambda}$ is the cardinality of the set of functions from $\lambda$ to $\kappa$ (this notation also doing double duty as the cartesian power); (iii) $\exp (\kappa)=2^{\kappa}, \exp ^{2}(\kappa)=\exp (\exp (\kappa))$, etc.; and (iv) $\kappa^{<\lambda}=\sup \left\{\kappa^{\alpha}: \alpha<\lambda\right\}$.

1.7 Theorem. (i) Suppose $A^{I}$ and $A^{J}$ are both $\kappa$-free. Then they are isomorphic $\left(A^{I} \cong A^{J}\right)$.

(ii) Suppose $A$ is nontrivial and $A^{I}$ is $\kappa$-free. Then $|I| \leq|A|^{\kappa}$.

Proof. (i) Let $P=\left\{p_{\xi}: \xi<\kappa\right\}$ (resp. $Q=\left\{q_{\xi}: \xi<\kappa\right\}$ ) be an enumeration of a pseudobasis for $A^{I}$ (resp. $\left.A^{J}\right)$. Using 1.1, there is, for each $j \in J$, a homomorphism $\varphi_{j}: A^{I} \rightarrow A$ taking $p_{\xi}$ to $q_{\xi}(j), \xi<\kappa$. Thus there is a homomorphism $\varphi: A^{I} \rightarrow A^{J}$ taking $p_{\xi}$ to $q_{\xi}, \xi<\kappa$. Similarly there can be found a homomorphism $\psi: A^{J} \rightarrow A^{I}$ taking $q_{\xi}$ to $p_{\xi}, \xi<\kappa$. Because $P$ and $Q$ are pseudobases, and $(\psi \circ \varphi) \mid P=\mathrm{id}_{P}$ and $(\varphi \circ \psi) \mid Q=\mathrm{id}_{Q}$, we know $\varphi$ and $\psi$ are mutually inverse isomorphisms.

(ii) Let $P$ be as above, and suppose $a \in A$ is such that there are distinct $i, j \in \bigcap_{\xi<\kappa} p_{\xi}^{-1}(a)$. Because $A$ is nontrivial, the projection maps $\pi_{i}$ and $\pi_{j}$ are distinct; whence $\pi_{i}\left|P \neq \pi_{j}\right| P$. But for any $\xi<\kappa, \pi_{i}\left(p_{\xi}\right)=p_{\xi}(i)=a=$ $p_{\xi}(j)=\pi_{j}\left(p_{\xi}\right)$. This contradiction forces the conclusion that for all $a \in A$, $\left|\bigcap_{\xi<\kappa} p_{\xi}^{-1}(a)\right| \leq 1$.

Let $h: I \rightarrow A^{\kappa}$ be defined by the conditions $\pi_{\xi} \circ h=p_{\xi}, \xi<\kappa$. For $i \neq j$ in $I$, we know from the last paragraph that there is some $\xi<\kappa$ with $p_{\xi}(i) \neq p_{\xi}(j)$. Thus $h$ is one-one and $|I| \leq|A|^{\kappa}$.

The focus of the remainder of this section is a sharpening of 1.7(ii) to say that if $I$ is "very much larger" than $A$, then better estimates are available for the cardinalities of possible pseudobases for $A^{I}$. This brings us to results that fall under the rubric of "straddling a measurable cardinal".

Our basic references for the theory of ultrafilters and large cardinals are the texts [7] and [8]. Recall that an ultrafilter $D$ on a set $I$ is $\kappa$-complete if whenever $S \subseteq D$ and $|S|<\kappa$, then $\bigcap S \in D$. A cardinal $\mu$ is measurable if there is a $\mu$-complete nonprincipal ultrafilter on $\mu$. By this definition, $\omega$ is a measurable cardinal. ( $\kappa$ is called Ulam-measurable if there is an $\omega_{1}$-complete nonprincipal ultrafilter on $\kappa$; equivalently if there is some uncountable measurable cardinal $\mu$ with $\mu \leq \kappa$.) An infinite cardinal $\mu$ is strongly compact if for every set $I$, every $\mu$-complete filter on $I$ can be extended to a $\mu$-complete ultrafilter on $I$. Clearly strongly compact cardinals are measurable, and $\omega$ is strongly compact. Measurable cardinals are "large"; i.e., they are regular and strongly inaccessible. (So if $\mu$ is measurable with $\kappa, \lambda<\mu$, then $\kappa^{\lambda}<\mu$.) Finally, it is consistent with the ZFC axioms of set theory that there are no uncountable measurable cardinals at all. (Gödel's universe $L$ of constructible 
sets contains no uncountable measurable cardinals, by a celebrated theorem of D. Scott.)

We identify the points of $I$ with the principal ultrafilters on $I$. The set $\beta(I)$ of all ultrafilters on $I$ is thus viewed as a superset of $I$, and is endowed with the Stone space topology: for each $J \subseteq I, J^{*}=\{D \in \beta(I): J \in D\}$. The sets $J^{*}, J \subseteq I$, form a "clopen" set basis for the Stone-Čech compactification of the discrete space $I$. For each $\kappa \geq \omega$, we let $\beta_{\kappa}(I)$ be the subspace of $\kappa$-complete ultrafilters on $I . \beta_{\omega}(I)=\beta(I)$; of course if $\kappa>|I|$, then $\beta_{\kappa}(I)=I$.

1.8 Lemma (Theorem 8.32 in [8]). If $\mu$ is strongly compact, $\mu \leq|I|$, and $|I|=|I|^{<\mu}$, then $\left|\beta_{\mu}(I)\right|=\exp ^{2}(|I|)$.

1.9 Remark. In the case $\mu=\omega$ in 1.8 , the condition $|I|=|I|^{<\omega}$ is redundant, and this result reduces to a famous theorem of B. Pospišil.

In the next theorem, some topological notions come into use. In particular, if $X$ is a topological space, the weight $w(X)$ of $X$ is the smallest infinite cardinal $\kappa$ such that $X$ has an open basis of cardinality $\leq \kappa$. Also, if $I$ is an index set, we endow $X^{I}$ with the (Tichonov) product topology: typical subbasic open sets are of the form $\pi_{i}^{-1}(U)$, where $i \in I$ and $U \subseteq X$ is (basic) open.

1.10 Theorem. (i) Suppose $A$ is a nontrivial finite algebra, $I$ is an infinite set, and $A^{I}$ is $\lambda$-free. Then $\lambda=\exp (|I|)$.

(ii) Suppose $A$ is a nontrivial algebra, I is a set such that there is some strongly compact cardinal $\mu$ with $|A|<\mu \leq|I|=|I|^{<\mu}$, and $A^{I}$ is $\lambda$-free. Then $\exp (\lambda)=\exp ^{2}(|I|)$.

Proof. (i) Let $A$ and $I$ be as hypothesized, with $P \subseteq A^{I}$ a pseudobasis of cardinality $\lambda$. By 1.7 (ii), $\lambda$ is infinite. For each $D \in \bar{\beta}(I)$ and $f \in A^{I}$, there is a unique $a \in A$ with $f^{-1}(a) \in D$. (Because $A$ is finite, every $f \in A^{I}$ is $D$-constant.) Let $D-\lim (f)$ be this unique $a \in A$. Then, since all the algebraic operations in $\Omega$ are finitary, $D$ - $\lim \in \operatorname{Hom}\left(A^{I}, A\right)$. Now suppose $E \in \beta(I)$ is different from $D$. Then there is some $J \subseteq I$ with $J \in D$ and $I \backslash J \in E$. Since $A$ is nontrivial,there is some $f \in A^{I}$ with $D-\lim (f) \neq E-\lim (f)$; so $D$ - $\lim \neq E-\lim$. Since $P$ is a pseudobasis, $D-\lim |P \neq E-\lim | P$.

Let $\eta: \beta(I) \rightarrow A^{P}$ be the assignment $D \mapsto D$-lim $\mid P$. Then $\eta$ is a one-one function. When $A$ has the discrete topology, making the power $A^{P}$ into a zerodimensional compact Hausdorff space, $\eta$ is also continuous. Indeed, if $\pi_{p}^{-1}(a)$ is a typical subbasic open subset, then $\eta(D) \in \pi_{p}^{-1}(a)$ if and only if $D-\lim (p)=$ $a$ if and only if $p^{-1}(a) \in D$. Thus $\eta^{-1}\left(\pi_{p}^{-1}(a)\right)=\left(p^{-1}(a)\right)^{*}$, a basic open subset of $\beta(I)$. Since $\eta$ is a continuous one-one map from a compact space to a Hausdorff space, $\eta$ is a topological embedding. Thus $w(\beta(I)) \leq w\left(A^{P}\right)$. Now $w(\beta(I))=\exp (|I|)$ and $w\left(A^{P}\right)=\lambda$. Thus $\lambda \geq \exp (|I|)$. SInce $P \subseteq A^{I}$, we already have $\lambda \leq \exp (|I|)$. so equality holds.

(ii) Let $A, \mu$, and $I$ be as hypothesized, with $P$ a pseudobasis of cardinality $\lambda$. We note first that if $\lambda<\mu$, then $|A|^{\lambda}<\mu$ because $\mu$ is strongly inaccessible. Thus $|A|^{\lambda}<|I|$, contradicting 1.7(ii). Thus $\lambda \geq \mu$; in particular $\left|A^{P}\right|=|A|^{\lambda}=$ $\exp (\lambda)$. Also we have $\lambda \leq\left|A^{I}\right|$, so $\exp (\lambda) \leq \exp ^{2}(|I|)$. By 1.8 , it remains to establish a one-one map from $\beta_{\mu}(I)$ to $A^{P}$.

Let $D \in \beta_{\mu}(I)$. Since $\mu$ is measurable and $|A|<\mu$, every $f \in A^{I}$ is $D$-constant. We may establish the map $\eta$ as in (i) above, and the proof is complete. (N.B.: $\eta$ is still continuous, but $\beta_{\mu}(I)$ is no longer compact, and 
we have no reason to believe $\eta$ is an embedding. Thus an argument involving topological weight seems no longer available.)

1.11 Corollary. Suppose $A$ is a nontrivial algebra and $\kappa$ is a cardinal such that for some strongly compact cardinal $\mu$, we have $|A|<\mu \leq \kappa$. If $A \uparrow \kappa$ is $\lambda$-free, then $\exp (\lambda)=\exp ^{3}(\kappa)$. Moreover, if $\mu=\omega$, then $\lambda=\exp ^{2}(\kappa)$. In particular, $A \uparrow \kappa$ is not $\kappa$-free.

Proof. Here we set $I=A^{\kappa}$. Then $|I|^{<\mu}=|I|$ automatically, so we may apply 1.10 .

Given an $\Omega$-algebra $A$ and an index set $I$, we say that a subset $Q \subseteq A^{I}$ is continuous if there is a $T_{1}$ topology on $A$ and a compact topology on $I$ such that each $q \in Q$ is a continuous map.

1.12 Proposition. $\Pi$ is a continuous subset of $A \uparrow \kappa$.

Proof. Let $A$ have any compact $T_{1}$ topology (of which there are many, e.g., the cofinite topology), and let $A^{\mathcal{K}}$ have the product topology. Then $A^{\mathcal{K}}$ is compact by Tichonov's product theorem. All the projection maps are continuous in this setting.

1.13 Theorem. Suppose $A$ is a nontrivial algebra, and $I$ is a set such that for some measurable cardinal $\mu$, we have $|A|<\mu \leq|I|$. Then $A^{I}$ has no continuous pseudobasis.

Proof. Let $A, \mu$, and $I$ be given as above, with $Q \subseteq A^{I}$ a continuous subset. Let $D \in \beta_{\mu}(I)$ be nonprincipal. As in the proof of 1.10 (ii), every $f \in A^{I}$ is $D$-constant, so we have the induced $D$ - $\lim \in \operatorname{Hom}\left(A^{I}, A\right)$. Thus, for each $q \in Q, q^{-1}(D-\lim (q)) \in D$. Since $A$ has a $T_{1}$ topology, so singleton subsets are closed, and $q$ is continuous, we infer that each $q^{-1}(D-\lim (q))$ is closed in $I$ for $q \in Q$. Now $Z=\left\{q^{-1}(D-\lim (q)): q \in Q\right\}$, being a subset of $D$, has the finite intersection property. Since $Z$ is also a collection of closed subsets of a compact space, we know $\cap Z \neq \varnothing$; whence there is a principal ultrafilter $E$ on $I$ with $Z \subseteq E$. Since $E \neq D$, we know (from the proof of $1.10(\mathrm{i})$ ) that $E-\lim \neq D$ - $\lim$. On the other hand, $D-\lim |Q=E-\lim | Q$, so $Q$ cannot be a pseudobasis.

1.14 Corollary. Suppose $A$ is a nontrivial algebra, $\kappa$ is a cardinal, and there is a measurable cardinal $\mu$ such that $|A|<\mu \leq \kappa$. Then $\Pi$ is not a pseudobasis for $A \uparrow \kappa$.

In the "straddling-a-measurable-cardinal" theorems above, we were able to give set-theoretic conditions that force fairly severe limitations on what kinds of pseudobases can occur in $A^{I}$. Of course, as the paragraph preceding 1.6 shows, one can never prove $A^{I}$ is not minimally free in general. A very natural setting in which $A^{I}$ is always minimally free, no matter how $|A|$ and $|I|$ are related, is given in the following.

1.15 Example. Let $A$ be a vector space (over a division ring). Every pseudobasis is Marczewski independent, hence linearly independent. Pseudobases are clearly maximal linearly independent sets; hence they are vector space bases. Therefore the analysis of what can and cannot be a pseudobasis for the power $A^{I}$ becomes a matter of linear algebra. In particular, a pseudobasis for an infinite power $A^{I}$ must have the maximal possible cardinality, since it must 
generate $A^{I}$. Also, since only the trivial vector space is rigid, $\Pi$ can never be a pseudobasis for $A \uparrow \kappa$ when $|A|>1$. (Indeed, the dimension of $A \uparrow \kappa$ far exceeds $\kappa$, even in the finite case.)

In the next section, we enrich the algebraic setting to that of unital rings, particularly division rings. Measurable cardinals figure both in deciding when $\Pi$ is a pseudobasis for $A \uparrow \kappa$, as well as in forcing $A^{I}$ to have no pseudobasis at all.

\section{POWERS OF DIVISION RINGS}

In the present section we focus on powers of unital rings, especially division rings. (So we take $\Omega$ to be appropriate, say $\{+, \cdot, 0,1\}$.) From $\S 1$ we know that if $\Pi$ is to have a chance at being a pseudobasis for $A \uparrow \kappa$, we need for $A$ to be rigid (1.5) and for $\kappa$ not to be too much greater than $|A|(1.14)$.

1.2 Remarks. (i) R. Schutt [14] has done a lot of (so far unpublished) work on minimal freeness in unital rings. In particular he has proved special cases of 1.5 and 1.7 in the ring-theoretic context. Another interesting fact he has discovered is that $\mathbb{Z}_{n} \uparrow 1$ (where $\mathbb{Z}_{n}$ is the ring of integers modulo $n$ ) has pseudobasis $\Pi$ if and only if $n$ is a power of a prime. To see, for example, that $\mathbb{Z}_{6} \uparrow 1$ is not 1 -free at all, set $\mathbb{Z}_{6} \uparrow 1=\mathbb{Z}_{6}^{6}$, so that the identity map id is $\langle 0,1,2,3,4,5\rangle$. Then $\Pi=\{$ id $\}$. By 1.6 , if $\mathbb{Z}_{6} \uparrow 1$ is 1 -free then $\Pi$ is a pseudobasis. Now $\varphi=\pi_{0}$ takes id to 0 ; so also does the (unital ring) homomorphism $\psi=3 \pi_{0}+4 \pi_{3}$. Since $\varphi \neq \psi$, we know $\mathbb{Z}_{6} \uparrow 1$ cannnt have $\Pi$ for a pseudobasis. That $\mathbb{Z}_{p} \uparrow 1$ is 1 -free when $p$ is a prime number follows from 2.2 below.

(ii) From here on in this section, our concern lies with powers of rigid division rings. The rational field $\mathbb{Q}$ and the real field $\mathbb{R}$ are well known to be rigid, as are the fields $\mathbb{Z}_{p}$, where $p$ is a prime. (Every finite division ring is a field, and every finite rigid field is some $\mathbb{Z}_{p}$. .) P. Pröhle [13] has shown that every field of characteristic zero embeds in a rigid field, so there are plenty of rigid fields.

(iii) No division ring is $\kappa$-free for $\kappa>0$, since division ring homomorphisms are embeddings.

(iv) Every product of division rings is a unital ring that is (von Neumann) regular; every regular unital ring whose idempotents are central is a product of division rings.

Our main positive result is the following.

2.2 Theorem. Let $A$ be a rigid division ring, and suppose $\kappa$ is a cardinal such that there is no measurable cardinal $\mu$ with $|A|<\mu \leq \kappa$. Then $\Pi$ is a pseudobasis for $A \uparrow \kappa$.

Proof. In view of 1.2, it suffices to show that every homomorphism $\varphi \in$ $\operatorname{Hom}(A \uparrow \kappa, A)$ is determined by its restriction $\varphi \mid \Pi$. So let $\varphi$ be given, and let $K_{\varphi}$ be the kernel ideal of $\varphi$. Now $\varphi \circ \delta=\mathrm{id}_{A}$ since $A$ is rigid, so $\varphi$ is onto. Consequently $K_{\varphi}$ is a maximal ideal in the ring $A \uparrow \kappa$. Since $\varphi$ preserves 1 , $K_{\varphi}$ is also proper. Let $I=A^{\kappa}$, and write $A \uparrow \kappa$ as $A^{I}$. It is a well-known result of A. Daigneault (see Exercise 4.1.30 in [7]) that $D_{\varphi}=\left\{k^{-1}(0): k \in K_{\varphi}\right\}$ is an ultrafilter on $I$; moreover this correspondence is a bijection between the proper maximal ideals of $A^{I}$ and the points of $\beta(I)$. For each $\xi<\kappa$, let $a_{\xi}=\varphi\left(\pi_{\xi}\right)$. Then $\pi_{\xi}-\delta\left(a_{\xi}\right) \in K_{\varphi}$ for all $\xi$, so $\left(\pi_{\xi}-\delta\left(a_{\xi}\right)\right)^{-1}(0)=\pi_{\xi}^{-1}\left(a_{\xi}\right) \in D_{\varphi}$. Let 
$Z=\left\{\pi_{\xi}^{-1}\left(a_{\xi}\right): \xi<\kappa\right\}$. Then $\bigcap Z=\{\vec{a}\}$, where $\vec{a}=\left\langle a_{\xi}: \xi<\kappa\right\rangle$. Now $\varphi \circ \delta=\mathrm{id}_{A}$ is the canonical embedding of $A$ into the $D_{\varphi}$-ultrapower; thus every $f \in A^{I}$ is $D_{\varphi}$-constant (i.e., $\varphi=D_{\varphi}$-lim in the notation of 1.10). We wish to show $D_{\varphi}$ is the principal ultrafilter $\{J \subseteq I: \vec{a} \in J\}$. For then $D_{\varphi}$, hence $K_{\varphi}$, is determined by $\varphi \mid \Pi . \varphi$ is thus determined, because if $\psi \in \operatorname{Hom}\left(A^{I}, A\right)$ has kernel $K_{\varphi}$, then $\psi$ too is onto since $A$ is rigid. Let $\theta \in \operatorname{Hom}\left(A^{I}, A^{I} / K_{\varphi}\right)$ be the natural quotient map. Then the induced homomorphisms $\varphi^{\prime}, \psi^{\prime} \in \operatorname{Hom}\left(A^{I} / K_{\varphi}, A\right)$ are isomorphisms and $\varphi=\varphi^{\prime} \circ \theta$, $\psi=\psi^{\prime} \circ \theta$. Because $A$ is rigid, we have $\varphi^{\prime}=\psi^{\prime}$; whence $\varphi=\psi$.

To show $D_{\varphi}$ is principal, assume otherwise. Then there is a largest cardinal $\mu$ such that $D_{\varphi}$ is $\mu$-complete, and this $\mu$ is measurable. If $\kappa<\mu$, then $\kappa^{+}<\mu$ (by inaccessibility); whence $\bigcap Z=\{\vec{a}\}$, an intersection of $\kappa$ members of $D_{\varphi}$, is a member of $D_{\varphi}$, and $D_{\varphi}$ is principal. Thus $\kappa \geq \mu$. By our hypothesis of "nonstraddling", we have $|A| \geq \mu$ also. Now $D_{\varphi}$ is $\mu^{+}$-incomplete, so there is a partition of $I$ into $\mu$ pieces such that no piece is in $D_{\varphi}$. This gives rise to a function $f \in A^{I}$ that is not $D_{\varphi}$-constant. This is a contradiction, and $D_{\varphi}$ is therefore principal.

2.3 Corollary. Let $A$ be a rigid division ring, $n<\omega$. Then $\Pi$ is a pseudobasis for $A \uparrow n$.

2.4 Remarks. (i) R. Schutt [14] proved 2.3 independently for the case $A$ is a field and $n=1$.

(ii) E. Fried and J. Sichler [10] have shown that there are arbitrarily large commutative unital rings that are rigid. By work in [2] and [12], this result was extended to show there are arbitrarily large $\kappa$-free commutative unital rings for any fixed $\kappa$. Pröhle's result [13] that there are arbitrarily large rigid fields can now be combined with 2.2 to show that there are arbitrarily large $\kappa$-free regular commutative unital rings for any fixed $\kappa$.

The "algebraic" part of the proof of 2.2 can be used to prove the minimal freeness of certain subrings of $A \uparrow \kappa$.

Let $A$ be an $\Omega$-algebra, $\kappa$ a cardinal. A subalgebra $B$ of $A \uparrow \kappa$ is called a $C$-subalgebra if: (i) $\Pi \cup \Delta \subseteq B$; and (ii) whenever $g \in B$ and $f_{\xi} \in B$ for $\xi<\kappa$, then $h \in B$, where $h(\vec{a})=g\left(\left\langle f_{\xi}(\vec{a}): \xi<\kappa\right\rangle\right)$.

2.5 Examples. (i) $\langle\Pi \cup \Delta\rangle$ is a $C$-subalgebra of $A \uparrow \kappa$ [2], as is $A \uparrow \kappa$ itself.

(ii) Let $\mathbb{R}^{\kappa}$ be given the usual Tichonov product topology, with $B \subseteq \mathbb{R} \uparrow \kappa$ the ring of continuous real-valued functions on $\mathbb{R}^{\kappa}$. Then $B$ is a $C$-subring of $A \uparrow \kappa$.

(iii) If $n<\omega$ and $B \subseteq \mathbb{R} \uparrow n$ is the ring of infinitely partially differentiable real-valued functions on $\mathbb{R}^{n}$, where all partial derivatives are continuous, then $B$ is a $C$-subring of $\mathbb{R} \uparrow n$.

Define a division ring $A$ to be $n$-formally real, $n<\omega$, if whenever $\sum_{m<n} a_{m}^{2}$ $=0$ in $A$, then $a_{m}=0$ for all $m<n$. If $f \in A^{I}$ is a unit of $A^{I}$, then $f$ has a unique multiplicative inverse denoted $1 / f$. Also, if $i \in I$, we let $\chi_{i} \in A^{I}$ be the map that takes $i$ to 1 and everything else in $I$ to 0 .

2.6 Theorem. Let $A$ be a rigid division ring, $n<\omega$, and $B \subseteq A \uparrow n$ a $C$ subring such that $1 / f \in B$ whenever $f \in B$ is a unit of $A \uparrow n$. Then $\Pi$ is a 
pseudobasis for $B$ provided either: (a) $A$ is $(n+1)$-formally real; or (b) $A$ is $n$-formally real and $\chi_{\vec{a}} \in B$ for all $\vec{a} \in A^{n}$.

Proof. By the discussion leading to 1.2 (and its proof), $\Pi$ is always an extendible subset of $B$ whenever $B \subseteq A \uparrow n$ is a $C$-subalgebra. Thus, to see that $\Pi$ is a pseudobasis for $B$, it suffices to check that every $\varphi \in \operatorname{Hom}(B, A)$ is determined by $\varphi \mid \Pi$. Proceed as in the proof of 2.2. Since $A$ is rigid, we know $\varphi$ is surjective and its kernel $K_{\varphi}$ is a proper maximal ideal of $B$. Let $a_{m}=\varphi\left(\pi_{m}\right), m<n$. For each $m<n, \pi_{m}-\delta\left(a_{m}\right) \in K_{\varphi}$, and $\left(\pi_{m}-\delta\left(a_{m}\right)\right)^{-1}(0)=\pi_{m}^{-1}\left(a_{m}\right)$. Let $s=\sum_{m<n}\left(\pi_{m}-\delta\left(a_{m}\right)\right)^{2}$, a sum of $n$ squares. Then $s^{-1}(0)=\bigcap_{m<n} \pi_{m}^{-1}\left(a_{m}\right)=\{\vec{a}\}$, where $\vec{a}=\left\langle a_{0}, \ldots, a_{n-1}\right\rangle$, since $A$ is $n$-formally real. Assume first that $A$ is $(n+1)$-formally real. Let $f \in K_{\varphi}$ and assume $f(\vec{a}) \neq 0$. Then $g=s+f^{2} \in K_{\varphi}$, a sum of $n+1$ squares; hence $g^{-1}(0)=\varnothing$. Thus $g$ is a unit of $A \uparrow n$ contained in $B$, so $1 / g \in B$. Since $g \in K_{\varphi}$, we infer that $1=\frac{1}{g} \cdot g \in K_{\varphi}$, so $K_{\varphi}$ is not proper. This contradiction tells us that every $f \in K_{\varphi}$ takes $\vec{a}$ to 0 ; whence $K_{\varphi} \subseteq\{f \in B: f(\vec{a})=0\}$. Since $K_{\varphi}$ is maximal proper ideal, equality must hold. Thus $K_{\varphi}$, hence $\varphi$, is determined by $\varphi \mid \Pi$.

Next assume $A$ is $n$-formally real and that $\chi_{\vec{a}} \in B$ for all $\vec{a} \in A^{n}$. We let $f \in K_{\varphi}$ and assume $f(\vec{a}) \neq 0$, where $a_{m}=\varphi\left(\pi_{m}\right), m<n$. Now $\chi_{\vec{a}} \in B$, so $g=\chi_{\vec{a}} f \in K_{\varphi}$, and $g^{-1}(0)=A^{n} \backslash\{\vec{a}\}$. Thus $(g+s)^{-1}(0)=\varnothing$, so $g+s \in K_{\varphi}$ is a unit of $A \uparrow n$. This tells us again that $K_{\varphi}=\{f \in B: f(\vec{a})=0\}$, and the proof is complete.

To end the section on a negative note, we show that when $A$ is a rigid division ring and $I$ is much larger than $A$, then $A^{I}$ is not minimally free. The proof depends on some topological aspects of $\beta_{\kappa}(I)$, the space of all $\kappa$-complete ultrafilters on $I$, as introduced just prior to 1.8 .

It is easy to see that if $I_{1}$ and $I_{2}$ are sets, with $f: I_{1} \rightarrow I_{2}$ any function, there is a unique continuous $\varphi: \beta_{\kappa}\left(I_{1}\right) \rightarrow \beta_{\kappa}\left(I_{2}\right)$ extending $f$. For $D \in \beta_{\kappa}\left(I_{1}\right)$, just let $\varphi(D)=\left\{J \subseteq I_{2}: f^{-1}(J) \in D\right\}$. (This also works when $f$ is the inclusion map $I_{1} \subseteq I_{2}$. Then $\varphi(D)=\left\{J \subseteq I_{2}: J \cap I_{1} \in D\right\}$.) We need a strengthening of this.

2.7 Lemma. Every $\kappa$-complete ultrafilter on the space $\beta_{\kappa}(I)$ converges.

Proof. Let $\mathscr{U}$ be a $\kappa$-complete ultrafilter on $\beta_{\kappa}(I)$. Then $\mathscr{V}=\{V \subseteq \beta(I)$ : $\left.V \cap \beta_{\kappa}(I) \in \mathscr{U}\right\}$ is a $\kappa$-complete ultrafilter on $\beta(I)$, and $\mathscr{V}$ converges to some (unique) $D \in \beta(I)$. We show $D \in \beta_{\kappa}(I)$, so $\mathscr{U}$ converges to $D$. Let $\lambda<\kappa$, with $J_{\xi} \in D$ for all $\xi<\lambda$. Let $J=\bigcap_{\xi<\lambda} J_{\xi}$. For each $\xi<\lambda, J_{\xi}^{*}$ contains some member of $\mathscr{V}$, so $\left(J_{\xi}^{*}\right)_{\kappa}=J_{\xi}^{*} \cap \beta_{\kappa}(I)$ contains some member $U_{\xi} \in \mathscr{U}$. Now $\left(J^{*}\right)_{\kappa}=\bigcap_{\xi<\lambda}\left(J_{\xi}^{*}\right)_{\kappa}$. If $J \notin D$, then $I \backslash J \in D$; and there is some $U \in \mathscr{U}$ with $(I \backslash J)_{\kappa}^{*}$ containing $U$. But $\left(J^{*}\right)_{\kappa} \supseteq \bigcap_{\xi<\lambda} U_{\xi} \in \mathscr{U}$, a contradiction. Thus $J \in D$, and so $D \in \beta_{\kappa}(I)$. Therefore $\mathscr{U}$ converges to $D$.

2.8 Lemma. Let $I_{1}, I_{2}$ be sets. Then every function from $I_{1}$ to $\beta_{\kappa}\left(I_{2}\right)$ extends uniquely to a continuous map from $\beta_{\kappa}\left(I_{1}\right)$ to $\beta_{\kappa}\left(I_{2}\right)$.

Proof. Let $f: I_{1} \rightarrow \beta_{\kappa}\left(I_{2}\right)$ be given. For each $D \in \beta_{\kappa}\left(I_{1}\right)$, define $\mathscr{U}_{D}=\{U \subseteq$ $\left.\beta_{\kappa}\left(I_{2}\right): f^{-1}(U) \in D\right\}$. Then $\mathscr{U}_{D}$ is a $\kappa$-complete ultrafilter on $\beta_{\kappa}\left(I_{2}\right)$, so by 2.7, $\mathscr{U}_{D}$ converges to some ultrafilter in $\beta_{\kappa}\left(I_{2}\right)$, which we label $\varphi(D)$. Clearly 
$\varphi$ extends $f$. If $J \subseteq I_{2}$, then $\varphi^{-1}\left(\left(J^{*}\right)_{\kappa}\right)=\left(\left(f^{-1}\left(\left(J^{*}\right)_{\kappa}\right)\right)^{*}\right)_{\kappa}$. Thus $\varphi$ is continuous.

2.9 Theorem. Let $A$ be a rigid division ring, with $I$ a set such that there is a measurable cardinal $\mu$ with $|A|<\mu \leq|I|$. Then $A^{I}$ is not minimally free.

Proof. Let $A, I$, and $\mu$ be as hypothesized, with $P$ a pseudobasis for $A^{I}$. Proceed as in the proof of 1.10(ii), assuming that $\mu$ is the least measurable cardinal with $|A|<\mu$. For each $D \in \beta_{\mu}(I)$, we have $D$-lim $\in \operatorname{Hom}\left(A^{I}, A\right)$; hence we let $\eta: \beta_{\mu}(I) \rightarrow A^{P}$ take $D \in \beta_{\mu}(I)$ to $D$ - $\lim \mid P . \eta$ is one-one and continuous; we now show $\eta$ is onto $A^{P}$.

Given $f \in A^{P}, f$ extends uniquely to a homomorphism $\varphi \in \operatorname{Hom}\left(A^{I}, A\right)$, since $P$ is a pseudobasis. Because $A$ is rigid, $\varphi$ is onto; hence its kernel $K_{\varphi}$ is a proper maximal ideal (as in the proof of 2.2). Moreover, $\varphi$ is the only homomorphism to $A$ with $K_{\varphi}$ as kernel. By Daigneault's correspondence, $D_{\varphi}=\left\{k^{-1}(0): k \in K_{\varphi}\right\}$ is an ultrafilter on $I$ for which every member of $A^{I}$ is $D_{\varphi}$-constant. It is clear that $f=\eta\left(D_{\varphi}\right)$ once we show $D_{\varphi} \in \beta_{\mu}(I)$. If $D_{\varphi}$ is principal, we are done. Otherwise, there is a largest cardinal $\nu$, a measurable cardinal, such that $D_{\varphi}$ is $\nu$-complete. But every $f \in A^{I}$ is $D_{\varphi}$-constant, so $|A|<\nu$. By the minimality condition on $\mu, \mu \leq \nu$; whence $D_{\varphi} \in \beta_{\mu}(I)$.

We now have a continuous bijection $\eta: \beta_{\mu}(I) \rightarrow A^{P}$, where $A$ has the discrete topology and $A^{P}$ has the product topology. (In case $\mu=\omega$, the proof has an easy finish: $\eta$ is a homeomorphism, since $\beta(I)$ is compact and $A^{P}$ is Hausdorff. But $\beta(I)$ has isolated points and $A^{P}$ does not. Contradiction.)

Now choose $D \in \beta_{\mu}(I)$ nonprincipal and $E \in \beta_{\mu}(I)$ principal (so $D$ is nonisolated and $E$ is isolated in $\beta_{\mu}(I)$ ). Then $A^{P}$ is a point-homogeneous space, so there is a homeomorphism $\psi$ on $A^{P}$ taking $\eta(D)$ to $\eta(E)$. Let $\theta=\psi \circ \eta$. By 2.8 there is a continuous $\rho: \beta_{\mu}(I) \rightarrow \beta_{\mu}(I)$ extending the function $\eta^{-1} \circ(\theta \mid I)$. Then $\theta$ and $\eta \circ \rho$ are continuous maps from $\beta_{\mu}(I)$ to $A^{P}$, and they agree on $I$. Since $I$ is dense in $\beta_{\mu}(I)$, we have $\theta=\eta \circ \rho$, i.e., $\psi \circ \eta=\eta \circ \rho$. Clearly $\rho$ is one-one and takes $D$ to $E$. But $E$ is isolated in $\beta_{\mu}(I)$, and this forces $D$ to be isolated. This is a contradiction, and we conclude that there can be no pseudobasis in $A^{I}$.

From 2.2 and 2.9 we immediately get the following.

2.10 Corollary. Let $A$ be a rigid division ring, $\kappa$ a cardinal. The following are equivalent:

(i) $\Pi$ is a pseudobasis for $A \uparrow \kappa$.

(ii) $A \uparrow \kappa$ is $\kappa$-free.

(iii) $A \uparrow \kappa$ is minimally free.

(iv) There is no measurable cardinal $\mu$ such that $|A|<\mu \leq \kappa$.

2.11 Remark. It is ironic that the rigidity of the base division ring is called for in the hypothesis of both 2.2 and 2.9. This assumption is absolutely essential for 2.2, by 1.5; and it seems indispensable in 2.9 at the point where we need to show $\eta: \beta_{\mu}(I) \rightarrow A^{P}$ is onto. We have made several attempts at circumventing rigidity; but have failed, even in the (minimally problematic) case $A$ is finite and $I$ is infinite. 


\section{Powers OF Boolean RINGS}

The situation with Boolean rings offers a sharp contrast to that with division rings, as regards minimal freeness. This is mainly due to Stone duality, linking the category of Boolean rings and unital ring homomorphisms contravariantly with the category of zero-dimensional compact Hausdorff spaces and continuous maps. The following consequence of this duality is a corollary of Theorem 3.5 in [1].

3.1 Theorem. Let $A$ be a Boolean ring, $\kappa$ a cardinal. Then $A$ is $\kappa$-free if and only if $A$ is the free Boolean ring on $\kappa$ generators.

Thus the problem of deciding whether a power $A^{I}$ is minimally free hinges on recognizing when direct powers of Boolean rings are free Boolean rings. This is not always an easy task; what we can show with the aid of Stone duality, together with well-known topological facts about certain Stone spaces, is the following.

3.2 Theorem. (i) Let $A$ be a Boolean ring, $I$ a nonempty set. Then $A^{I}$ is not minimally free if either: (a) $I$ is uncountable; or (b) $|I|=\omega$ and $|A|<|A|^{\omega}$.

(ii) Let $A$ be a Boolean ring, $\kappa$ a cardinal. If $A \uparrow \kappa$ is minimally free, then $\kappa<\omega$. If, in addition, $A$ is finite, then $A \uparrow \kappa \cong \mathbb{Z}_{2} \uparrow n$, the free Boolean ring on $n$ generators, for some $n<\omega$.

Proof. (i) Let $\sigma(A)$ denote the Stone space of ultrafilters on the Boolean ring (lattice) $A$. Then $\sigma\left(A^{I}\right)$ is the $I$-indexed copower of $\sigma(A)$, namely the Stone-Čech compactification $\beta(\sigma(A) \times I)$ (where $I$ has the discrete topology). $\sigma(A) \times I$ is locally compact, and is hence embedded naturally as an open subset of $\beta(\sigma(A) \times I)$. Thus if $I$ is uncountable, then $\beta(\sigma(A) \times I)$ possesses an uncountable family of pairwise disjoint nonempty open subsets. But the Stone space of a free Boolean ring is a generalized Cantor space, of the form $2^{\lambda}$, which has no such family. (Tichonov products of spaces satisfying the countable chain condition also satisfy this condition.) Consequently $A^{I}$ is not free; by $3.1, A^{I}$ is not minimally free.

Suppose now that $|A|<|A|^{\omega}$ and $|I|=\omega$. If $A^{I}$ is minimally free, hence free, it must have a free basis of cardinality $\lambda=|A|^{\omega}$. Its Stone space must therefore be $2^{\lambda}$. But for dyadic spaces, of which $2^{\lambda}$ is an example, the weight equals the character. Now $w\left(2^{\lambda}\right)=\lambda$; hence, because of homogeneity, each point of $2^{\lambda}$ has a neighborhood basis of cardinality $\lambda$, and no point has a neighborhood basis of smaller cardinality. But in $\sigma\left(A^{I}\right)=\beta(\sigma(A) \times I), \sigma(A)$ is embedded as an open subset. By Stone duality, $w(\sigma(A))=|A|$; whence $\sigma\left(A^{I}\right)$ contains points having neighborhood basis of cardinality $|A|<\lambda$. Thus $A^{I}$ is not free, hence not minimally free.

(ii) The Boolean rings $\mathbb{Z}_{2} \uparrow n, n<\omega$, are all free on $n$ generators (so indeed $\Pi$ is a pseudobasis). So suppose $A \uparrow \kappa$ is minimally free. Then $\kappa<\omega$ by (i) above. If $A$ is also finite, then so is $A \uparrow \kappa$; whence $A \uparrow \kappa \cong \mathbb{Z}_{2} \uparrow n$, where $n=\kappa \log _{2}(|A|)+\log _{2}\left(\log _{2}(|A|)\right)$.

\section{ACKNOWLEDGMENT}

The author would like to thank his colleagues Wim Ruitenburg and John Simms for several stimulating conversations and useful suggestions during the preparation of this paper. 


\section{REFERENCES}

1. P. Bankston and R. Schutt, On minimally free algebras, Canad. J. Math. 37 (1985), 963978.

2. P. Bankston, A note on large minimally free algebras, Algebra Universalis 26 (1989), 346350.

3. __ Minimal freeness and commutativity, Algebra Universalis 29 (1992), 88-108.

4. P. Bankston and R. A. McCoy, On the classification of minimally free rings of continuous functions, General Topology and Applications (Proc. 1988 Northeast Conf., R. M. Shortt, ed.), Dekker, New York, 1990, pp. 51-58.

5. _ H-enrichments of topologies, Topology Appl. 42 (1991), 37-55.

6. G. Birkhoff, On the structure of abstract algebras, Proc. Cambridge Philos. Soc. 31 (1935), 433-454.

7. C. C. Chang and H. J. Keisler, Model theory, North-Holland, Amsterdam, 1973.

8. W. W. Comfort and S. Negrepontis, The theory of ultrafilters, Springer-Verlag, Berlin, 1974.

9. M. Dugas, A. Mader, and C. Vinsohaler, Large E-rings exist, J. Algebra 108 (1987), 88101.

10. E. Fried and J. Sichler, Homomorphisms of commutative rings with unit elements, Pacific J. Math. 45 (1973), 485-491.

11. G. Grätzer, Universal algebra, 2nd ed., Springer-Verlag, New York, 1979.

12. I. Křiž and A. Pultr, Large k-free algebras, Algebra Universalis 21 (1985), 46-53.

13. P. Pröhle, Does a given subfield of characteristic zero imply any restriction to the endomorphism monoids of fields? Acta Math. 50 (1986), 15-38.

14. R. Schutt, (private communication).

Department of Mathematics, Statistics and Computer Science, Marquette UniverSity, Milwaukee, Wisconsin 53233

E-mail address: paulb@mscs.mu.edu 